\title{
Second International Workshop on Building Sustainable Open Source Communities OSCOMM 2010
}

\author{
Imed Hammouda $^{1}$, Timo Aaltonen ${ }^{1}$, and Andrea Capiluppi ${ }^{2}$ \\ ${ }^{1}$ Tampere University of Technology, Finland \\ \{imed.hammouda, timo.aaltonen\} atut.fi \\ ${ }^{2}$ University of East London, UK \\ a.capiluppi@uel.ac.uk
}

\begin{abstract}
The Second International Workshop on Building Sustainable Open Source Communities (OSCOMM 2010) aims at building a community of researchers and practitioners to share experiences and discuss challenges involved in building and maintaining open source communities.
\end{abstract}

\section{Workshop Scope}

Open source software is gaining momentum in several forms. In addition to the huge increase in the number of open source projects started and the remarkable rise of FLOSS adoption by companies, new models of participation in the movement are emerging rapidly. For instance, companies are increasingly releasing some of their proprietary software systems as open source on one hand and acquiring open source software on the other hand. For all these forms of involvement, a central question is how to build and maintain a sustainable community of users and developers around the open source projects.

Research findings show that developing and maintaining online communities in general is a complex activity. In the case of open source communities, the situation is worsened as the problem is multi-facet (e.g. legal, social, technical, business) bringing own kinds of challenges. We think that it is the right time for the research community and the industry to discuss the community building problem from its various perspectives by exchanging related experiences, sharing relevant concerns, and proposing guidelines to manage the challenges highlighted earlier. This is vital as more and more companies are moving towards community-driven development models.

\section{Workshop Theme}

Workshop topics include (but are not limited to):

- challenges of building and maintaining open source communities covering concerns related to legal, socio-cultural, business, technical, etc. dimensions;

- organization and interaction schemes in open source communities; 
- models and classification schemes of communities: participation (e.g. volunteer, mixed, company-based), origin (e.g. individual, company), host (e.g. academy, company), scope (e.g. public, corporate);

- practical approaches, best practices, frameworks, methodologies, technologies, tools, and environments to support community building and management;

- industrial involvement in building, managing and interfacing with communities: opening up software platforms and acquiring open source software, motives and cost-benefit models;

- building open source communities: the role of companies, academy, governments, NGOs, and individuals;

- open source communities versus other kinds of communities such as firm-hosted communities, corporate communities, social networks, global software teams;

- experience reports and lessons on building and maintaining open source communities.

\section{Workshop Goals}

The goal of the workshop is to bring together interested academics, practitioners, and enthusiasts to discuss topics related to open source communities. The workshop will offer an opportunity for the participants to share experiences and discuss challenges involved in building and maintaining open source communities. The workshop will also identify key research issues and challenges that lie ahead.

\section{Further Information}

Further information regarding organization and program of the workshop is available at http://tutopen.cs.tut.fi/oscomm10/. 